American Journal of Applied Sciences 6 (1): 187-193, 2009

ISSN 1546-9239

(C) 2009 Science Publications

\title{
Quality Evaluation of Reconstructed Biological Signals
}

\author{
${ }^{1}$ Mikhled Alfaouri, ${ }^{1}$ Khaled Daqrouq, ${ }^{1}$ Ibrahim N. Abu-Isbeih, ${ }^{2}$ Emad F. Khalaf, \\ ${ }^{1}$ Abdel-Rahman Al-Qawasmi and ${ }^{1}$ Wael Al-Sawalmeh \\ ${ }^{1}$ Department of Communication Engineering, \\ ${ }^{2}$ Department of Computer Engineering, \\ Philadelphia, University 19392, P.O. Box 1, Amman-Jordan
}

\begin{abstract}
This research presents the use of wavelet transforms for reconstructed signal evaluation for biological signals. The method adapted on this research, is a new modeling technique and based on calculating the reconstruction error of the wavelet transform sub-signals that have different frequency bands and different deformation degree. The paper processes a special set of application in biomedical field, called Electrocardiogram (ECG) signal. Compression applied on the electrocardiogram (ECG) and speech signals. Discrete Wavelet Transformation (DWT) with the thresholding compression method was applied to test the proposed reconstruction evaluation method. The proposed algorithm technique gave an excellent result showing that, using wavelet transform is a suitable technique for reconstruction signal evaluation. Also, the presented method determined the exact place and location of deformation in the reconstruction signal.
\end{abstract}

Key words: Quality, ECG signals, speech signal, data compression, wavelet transform, discrete wavelet transform

\section{INTRODUCTION}

As reported recently in literature, an increasing amount of interest of research and analysis of (ECG) has been conducted dealing with the data compression and reconstruction. The need of signal transmission over telephone lines or antenna for remote analysis, makes the compression and data reconstruction of the signal an important issue in signal processing and shed some light for researchers, many researchers were relatively reported about compression techniques and the methods were improved rapidly and gradually year after year ${ }^{[1,2]}$. An electrocardiogram (ECG) is a graphic display of the electrical activity of the heart. It provides essential information to the cardiologist to be used for the diagnostic purpose. The need of ECG signal compression exists in many transmitting of the ECG signal through telephone lines and storage applications via antennas ${ }^{[1]}$. Indeed, an excellent monographic paper on this subject, explains the save of a crucial time and unnecessary difficulties in the intensive coronary care unit, or in long term 24-48 $\mathrm{h}$ wearable monitoring tasks (Holter's device $)^{[3]}$.

Memory costs may render such a solid state Holter's device impractical. If efficient compression methods are employed, memory requirements may drastically drop to make the solid state high quality Holter device commercially feasible.
On another hand, speech signal is very important biological signal that should be compressed to accomplish less size. This assists greatly in storage and transmission needs.

But, the most important stage of the original signal is the reconstruction from of the compressed data. Many methods are used for signal reconstruction for compression techniques. Inverse Fast Fourier Transform (IFFT), Inverse Wavelet Transform (IWT) and the popular techniques, decoding methods for reconstruction. The reconstruction signal quality degree determines the utilities of the given compression technique. The compression method of bad reconstruction quality is pointed as not useful, even when high compression ratio is accomplished.

The most popular method, that is used for ECG signal reconstruction quality evaluation is the Percentage Root Mean Square Difference (PRD):

$$
\mathrm{PRD}=\sqrt{\frac{\sum_{\mathrm{n}=0}^{N-1}\left(\mathrm{f}(\mathrm{n})-\mathrm{f}^{\prime}(\mathrm{n})\right)^{2}}{\sum_{\mathrm{n}=0}^{N-1} f(n)^{2}}} * 100 \%
$$

where, $f(n)$ is the original ECG signal and $f^{\prime}(n)$ is the reconstructed ECG signal.

Corresponding Author: Dr. Mikhled Alfaouri, Department of Communication and Electronics Engineering, Philadelphia, University 19392, P. O. Box 1, Amman-Jordan 
Percentage root mean square difference (PRD) measures the square difference average between the original and reconstructed signals. This method determines the deformation percent in the built signal.

$\mathrm{II}^{[4]}$ new method for (ECG) signal reconstruction is presented, this method based on calculating distinct errors in particular places of (ECG) signal, the chosen places are know in physiology as P-wave, Q-wave, Rwave, $\mathrm{S}$-wave and T-wave as shown in Fig. 1. The error is calculated for each wave in amplitude and time duration. The errors are divided into two error simplified forms of original equations and are presented mathematically by:

$$
\mathrm{WAE}=\frac{\mathrm{WA}_{\mathrm{Org}}-\mathrm{WA}_{\mathrm{Rec}}}{\mathrm{WA}_{\mathrm{Org}}} 100 \%
$$

where, WAE is the wave amplitude error, $\mathrm{WA}_{\text {Org }}$ is the original wave amplitude and $\mathrm{WA}_{\text {rec }}$ is the reconstructed wave amplitude and the wave time error is:

$$
\mathrm{WTE}=\frac{\mathrm{WT}_{\text {Org }}-\mathrm{WT}_{\mathrm{Rec}}}{\mathrm{WT}_{\text {Org }}} 100 \%
$$

where, WTE is the wave time error, $\mathrm{WT}_{\text {Org }}$ is the original wave time and $\mathrm{WT}_{\text {rec }}$ is the reconstruction wave time.

Equation 2 and 3 calculate the relative errors, provides the relative deformation to original wave morphology. This approach needs very good detection technique of onset, offset and peak points of given wave. These points are known as fiducial points or characteristic points.

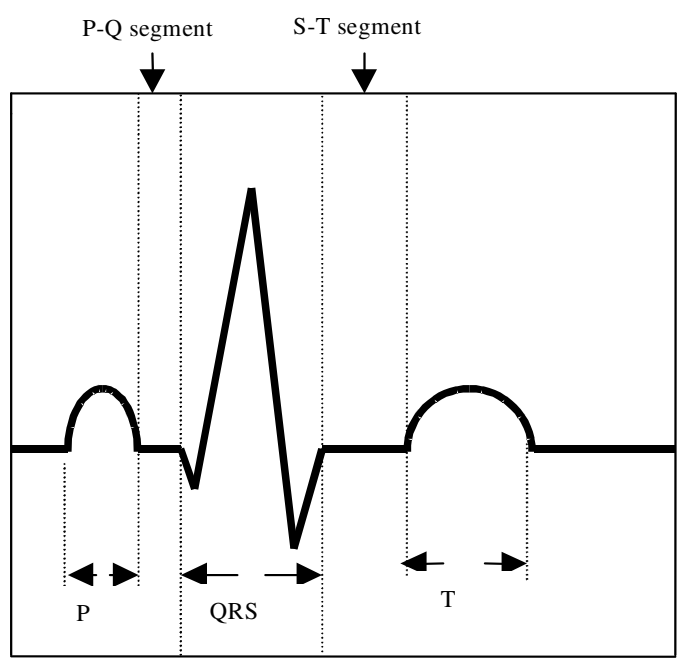

Fig. 1: A typical electrocardiogram
Also, percentage root mean square difference (PRD) measures the deformation in the whole signal, but this approach gives a superior measuring performance due to the exact error wave measuring in both time and amplitude domains. But the difficulties appear in fiducial point's detection of such nonstationary (ECG) signal, which varies over time rapidly very quickly. Therefore, in this research, a method that based on calculating the deformation of (DWT) coefficients of reconstruction signal, without the need of detection method as presented in ${ }^{[8]}$.

In speech signal field, the reconstruction signal is valuated using other techniques. The most popular methods that are used in speech reconstruction signal (FFT), Power Spectrum Density (PSD) and Signal-toNoise-Ratio (SNR):

$$
\operatorname{SNR}=10 \log _{10} \frac{\sum_{n=0}^{N-1}\left(f^{\prime}(n)\right)^{2}}{\sum_{n=0}^{N-1}\left(f(n)-f^{\prime}(n)\right)^{2}}
$$

where, $f(n)$ is original signal and $f^{\prime}(n)$ is speech reconstructed original signal.

The most essential evaluation method for speech signal evaluation is the objective method, which based on hearing the speech of the reconstruction signal by group of people.

The proposed method in this research is based on introducing a novel modeling technique using wavelet transformation for signal error evaluation with the use of (ECG) signal as one of the most important nostationary stochastic data applications in the biomedical digital signal processing field and biological nostationary stochastic speech signal as popular signal in communication.

\section{COMPRESSION METHOD}

The main goal for the use of the (ECG) signal compression is to achieve a minimum information rate, while retaining the relevant diagnostic results, in order to reserve all clinical information contained in the original signal.

Data compression techniques are used to categorize those of the compressed data to be reconstructed in order to form the original signal, also for higher compression ratio, the error is achieved in reconstructed signal. The effectiveness of an (ECG) compression technique is accomplished in terms of compression ratio (CR); which is the ratio of the size of the compressed data to that of the original data in the lossy 
compression method. The problem is more complicated for speech signal because of intensive data rate and information deformation sensitivity.

The efficient data compression may be achieved only for lossy compression techniques (which allowable for reconstruction error).

In this research, a method of transformation to data reconstruction is used. This method is based on discrete wavelet transformation compression in conjunction with the thresholding method using a set of wavelet functions, in order to decompose the (ECG) signal as a set of coefficients $^{[3]}$. The wavelet coefficients obtained from (DWT) corresponds to the measured data of the (ECG) also the speech components in intervals frequency bands.

A quick rehash of wavelet transform used in this research is presented in the following section:

Wavelet transform: A wavelet is simply a small wave which has energy concentrated in time to give a tool for the analysis of transient, nonstationary or time-varying phenomena such as a wave shown in Fig. 1.

A signal of the function $f(t)$ is shown in Fig. 2 which can often be better analyzed and expressed as a linear decomposition of sums: (products of the coefficient and function). As in Fourier series, one uses sine and cosine functions as orthogonal basis functions. But in the wavelet expansion, the two-parameter system is constructed such that one has a double sum and the coefficients with two indices. The set of coefficients are called the Discrete Wavelet Transform (DWT) of $f(t)$. Namely called a wavelet series expansion which maps a function of a continuous variable into a sequence of coefficients much of the same way as Fourier series dose with the main useful four properties:

The representation of singularities and the representation of local basis functions to make the algorithms adaptive in-homogeneities of the functions, to have the unconditional basis property for a variety of function classes to provide a wide range of information about the signal.

The representation of smooth functions defined as:

$$
\mathrm{f}=\sum_{\mathrm{m}, \mathrm{n}}\left(\mathrm{f}, \Psi_{\mathrm{m}, \mathrm{n}}\right) \bar{\Psi}_{\mathrm{m}, \mathrm{n}}
$$

In the wavelet transform defined $\mathrm{in}^{[4]}$, the original signal (1-D, 2-D, 3-D) is transformed using predefined wavelets. The wavelets are orthogonal, orthonormal, or biorthogonal, scalar or multiwavelets ${ }^{[10,11]}$. Then, we can write the continuous wavelet transform as follows:

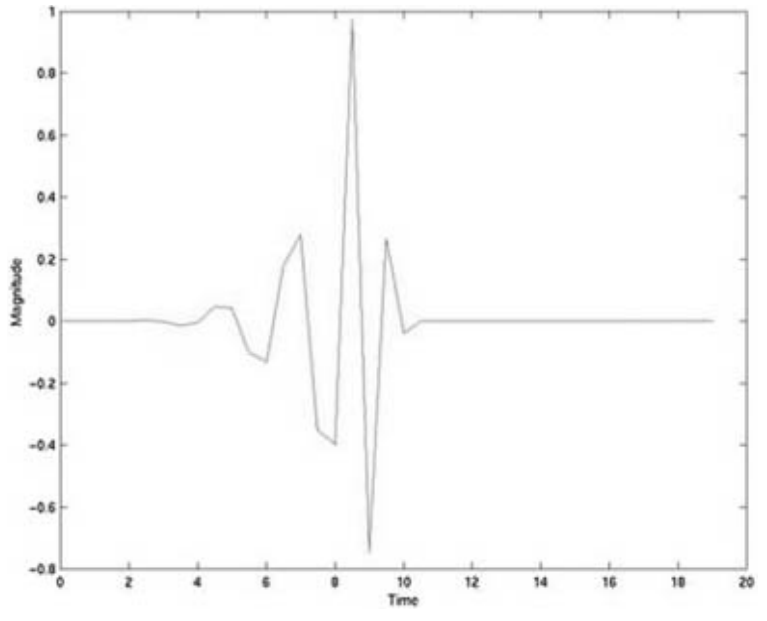

Fig. 2: Wavelet function

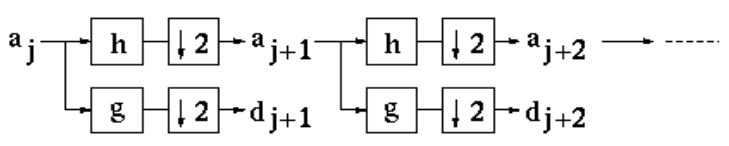

a)

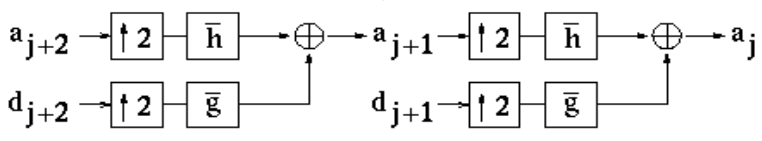

b)

Fig. 3: Filter bank tree of (a) Decomposition and (b) Reconstruction

$$
\left(T^{\text {wav }} f\right)(a, b)=|a|^{-1 / 2} \int f(t) \psi\left(\frac{t-b}{a}\right) d t
$$

with $\Psi$ is a function of used basis series.

The continuous inverse wavelet transform: In discrete case, the wavelet transform is modified to represent a filter bank tree using the decomposition/reconstruction shown in Fig. 3 and defined mathematically as:

$$
f=C_{\psi}^{-1} \iint \frac{1}{a^{2}}\left(T^{w a v} f\right)(a, b) \psi^{a, b} d a d b
$$

This process can be expressed completely in terms of the digital filters $g(n)$ and $h(n)$ as follows:

$$
\begin{aligned}
& d_{j+1}(p)=\sum_{n} g(n-2 p) \quad a_{j}(n) \\
& a_{j+1}(p)=\sum_{n} h(n-2 p) a_{j}(n)
\end{aligned}
$$


where, the set of numbers $a_{j}(n)$ represents the approximation of the signal at the resolution $2^{-j}$ and the set of numbers $d_{j}(n)$ represents the details lost in approximating the signal at resolution $2^{-(j-1)}$ at the resolution $2^{-\mathrm{j}}$.

Most applications of this result hinge on constructing wavelets such that a few non-zero coefficients proximate the signal, i.e. the detail sequence at higher resolutions is close enough to zero so as to be ignored.

Recently, new Techniques for dealing with compression are reported in literature as: Direct compression and Transform-based compression. A transform method has received lately much attention as a useful technique in the wavelet transform. The wavelet transform is a time-scale representation technique which utilizes correlation with the translations and dilations of wavelet functions. Applying the DWT to the tested signal. Lately, we have used the wavelet transform to analyze the signal. Moreover with regard to the translation operation allows signal features to be isolated in time, while the dilation operation allows features exiting at different scales to be identified.

In this research the compression algorithm using (DWT) to test presented method is presented, this part is implemented using the following steps:

DWT signal conversion: This decomposes the signal into subsignals of different pass bands frequencies as shown in Fig. 4.

Thresholding: Thresholding process is applied to the (DWT) coefficient values to compare signal energy with those of less than the threshold value and set them equal to zero. Mainly high pass frequency coefficients are thresholded.

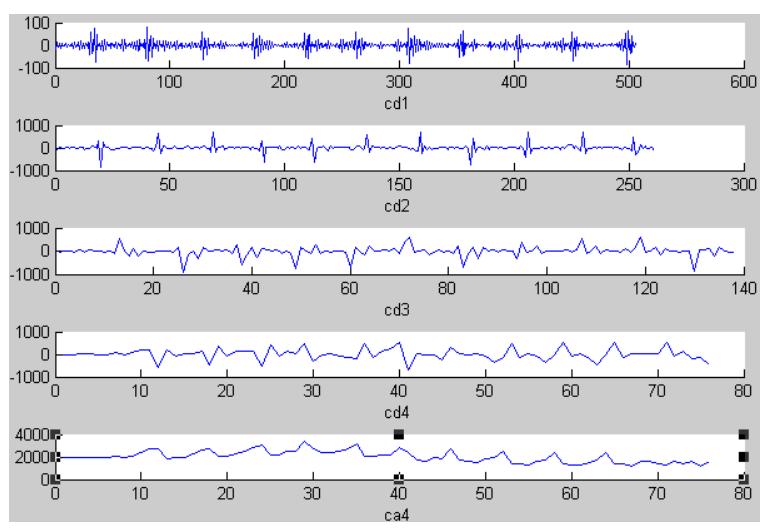

Fig. 4: Sub-signals of DWT of ECG signal $\left(\mathrm{d}_{1}, \mathrm{~d}_{2}, \mathrm{~d}_{3}, \mathrm{~d}_{4}, \mathrm{~s}_{4}\right)$
Coding: A simple coding scheme is used to code the thresholded (DWT) coefficients, such as run-length coding system or other to write the zeros by smallest size. Here we use zero-position method published before $^{[8]}$.

Signal reconstruction: Applying Inverse Discrete Wavelet Transform (IDWT) to reconstruct the (ECG) signal again. This is achieved by basically computing the convoluting of the signal with conjugate version of wavelet basis functions.

Errors analysis and conventional distortion measures: Finally, the critical quantities compression of the reconstructed signal and the original signal are obtained by the error expressions as shown in Fig. 5.

The algorithm is implemented using MATLAB.

\section{MATERIALS AND METHODS}

In this part of the research, the reconstructed signal is evaluated and analyzed using discrete wavelet transform. The method is based on computing (PRD) of wavelet sub-signals to determine the exact deformation places and locations in reconstruction signal.

The proposed algorithm presented in this research is a new technique for reconstruction signal evaluation and summarized in the following steps:

- Decomposing reconstructed and original signals into a discrete wavelet transform sub-signals $\mathrm{d}_{1}, \mathrm{~d}_{2}, \mathrm{~d}_{3}, \mathrm{~d}_{4}$ and $\mathrm{s}_{4}$, shown in Fig. 4

- Computing (PRD) of each sub-signal, (PRDS) is shown in Fig. 5

- Comparing between the (PRDS) of the original and the reconstructed signals with sub-signals:

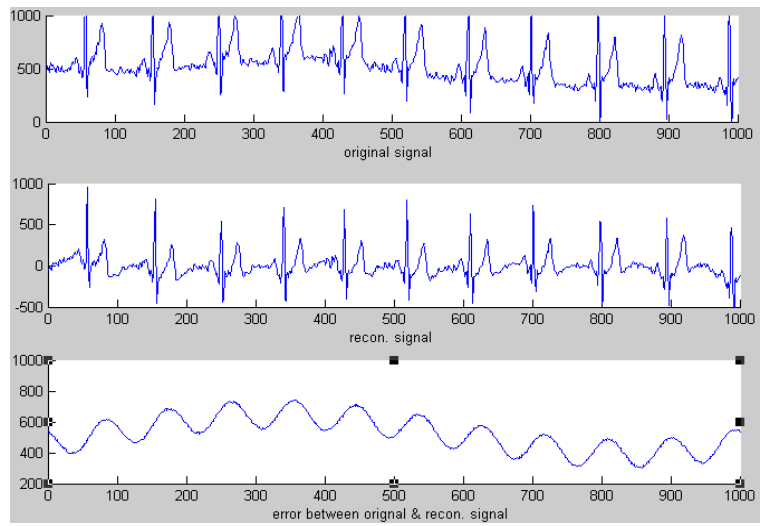

Fig. 5: The original and reconstructed signals with error $(\mathrm{CR}=2.45, \mathrm{PRD}=0.970)$ 
Am. J. Applied Sci., 6 (1): 187-193, 2009

$$
\operatorname{PRDS}=\sqrt{\frac{\sum_{n=0}^{N-1}\left(d_{\text {org J }}(n)-\left(d_{\text {rec J }}(n)\right)^{2}\right.}{\sum_{n=0}^{N-1}\left(d_{\text {org J }}(n)\right)^{2}}} * 100 \%
$$

where, $\mathrm{d}_{\text {org }}$ is the (DWT) detailed sub-signal of $\mathbf{J}$ level of original and signal $d_{\text {rec }}$ is the (DWT) detailed sub-signal of $\mathrm{J}$ level of reconstructed

- The most important part of presented evaluation method is Signal-M $\mathrm{PRD}_{\mathrm{PD}^{-}}$Ratio (SMR):

$$
\mathrm{SMR}=10 \log _{10} \frac{\sum_{\mathrm{n}=0}^{\mathrm{N}-1}\left(\mathrm{f}^{\prime}(\mathrm{n})\right)^{2}}{\mathrm{M}_{\mathrm{PRD}}}
$$

where, $\mathrm{M}_{\mathrm{PRD}}=\frac{\sum_{\mathrm{j}=1}^{\mathrm{J}} \mathrm{PRDS}(\mathrm{j})}{\mathrm{J}}$ and $\mathrm{J}$ is the DWT level.

\section{RESULTS AND DISCUSSION}

The method described appears to overcome the aforementioned evaluation compression techniques and provides vast amount of accurate, meaningful information of great diagnostic assistance of the ECG or speech signal.

ECG signal: From the information obtained using the compression of the (ECG) signal of over eleven different heart beat period, we set up (ECG) classification for several cases of threshold and quantization to give several (CR) and PRDS values. The threshold and the quantization change leads to different (CR), this can help in testing the given method using several cases of reconstruction signal, but at the expense of adding high pass noise of different amplitudes: 5\%, $15 \%$ or $30 \%$ of the peak of original signal. The high compression ratios are a sign of presence of deformation in reconstruction signal. This will affect PRDS value, which can be noticed in Table 1 and make the deformation is clear in the DWT sub-signals. The effect of the (CR) on error can be noticed in Fig. 4.

From literature survey, that highest acceptable PRD value of ECG is $8 \%$. The cardiologists have determined that more than $8 \%$ causes essential deformation in the information contained in the ECG morphology. It means huge changing of clinical information which is important for cardio logical diagnosis. It is good to start from this point to determine the deformation in the reconstruction signal of ECG compression methods. The same criteria is used for FFT compression method. In results part we use this criteria to test the presented method quality evaluation performance of ECG signal compression methods.
Table 1: Compression Ratios and PRD for Very high pass noise signal $30 \%$ of the peak value of original signal (WT compression method), where bold font presents the deformation places

\begin{tabular}{llrrrrr}
\hline $\mathrm{CR}$ & $\mathrm{PRD}_{\text {org }}$ & \multicolumn{1}{c}{$\mathrm{PRD}_{\mathrm{d} 1}$} & \multicolumn{1}{c}{$\mathrm{PRD}_{\mathrm{d} 2}$} & $\mathrm{PRD}_{\mathrm{d} 3}$ & $\mathrm{PRD}_{\mathrm{d} 4}$ & $\mathrm{PRD}_{\mathrm{d} 5}$ \\
\hline 2.2624 & 5.2210 & 02.9244 & 6.6003 & 8.8392 & 4.3616 & 0.4712 \\
4.5956 & 5.4473 & 130.0513 & 40.1934 & 9.5266 & 5.2102 & 0.4709 \\
6.0314 & 5.7173 & 152.4513 & 47.4734 & 10.8566 & 6.2822 & 0.4755 \\
6.8587 & 6.0560 & 172.6690 & 52.3315 & 12.1807 & 7.2971 & 0.4898 \\
7.3638 & 6.4273 & 194.6539 & 5.8150 & 13.4111 & 8.6282 & 0.5074 \\
8.4246 & 7.7880 & 262.3979 & 64.6029 & 16.5290 & 2.0305 & 0.5967 \\
9.3284 & 9.3307 & 328.8328 & 2.9699 & 19.4489 & 15.7807 & 0.7060 \\
\hline
\end{tabular}

Speech signal: The most important speech reconstruction quality assessment is objective method. This is based on hearing the reconstructed method by few of persons to determine the quality of the signal. In results part we use this criteria to test the presented method quality evaluation performance of speech signal compression methods.

The presented method was applied with (DWT) compression method (Table 1) and also with (FFT) compression method (Table 2). The biggest (PRDS) always contained in $d_{1}$, because of intensive size of data and existence of high pass noise.

The results in these tables show smaller magnitude of (PRDS) with (DWT) compression method. This provides that, this type of transforms is more suitable for the distinct nature of non-stationary signals. From the aforementioned results, the following specific detailed are concluded:

\section{ECG signal:}

- The (PRD) of original signal has the magnitude nearly equal to the average PRDS of $\mathrm{d}_{3}, \mathrm{~d}_{4}$ and $\mathrm{s}_{4}$, because these sub-signals have the most of original energy contained in the signal.

- Table 1 shows the (PRDS) results of wavelet compression method for different CR. The results show that deformation (the deformation places in Table 1 and 2 are denoted by bold font) exist in $\mathrm{d}_{1}$ and $\mathrm{d}_{2}$ for all original signal of (PRD) less than $8 \%$. But deformation places exist in $\mathrm{d}_{1}, \mathrm{~d}_{2}$ and $\mathrm{d}_{4}$ in not acceptable (PRD) $(9.33 \%)$ of (ECG) signal. The deformation in $\mathrm{d}_{4}$ leads to have essential deformation in reconstruction quality, because of most of signal energy exists in low bands of frequencies $\left(\mathrm{d}_{4}\right.$ and $\left.\mathrm{s}_{4}\right)$.

- For (FFT) compression method, we can notice that for acceptable (PRD) (0.97 or 5.19\%) of original signal, the deformation places exist in $\mathrm{d}_{1}$ and $\mathrm{d}_{2}$ but for not acceptable (more than 8\%) the deformation starts to be in $d_{4}$ that has big part of energy of the signal. Therefore, the bad quality in reconstruction signal is appeared. 
Am. J. Applied Sci., 6(1): 187-193, 2009

Table 2: Compression Ratios \& PRDS for very high pass noise signal 30\% of peak value of original signal (FFT compression method), where bold font presents the deformation places

\begin{tabular}{|c|c|c|c|c|c|c|}
\hline CR & $\mathrm{PRD}_{\text {org }}$ & $\mathrm{PRD}_{\mathrm{d} 1}$ & $\mathrm{PRD}_{\mathrm{d} 2}$ & $\mathrm{PRD}_{\mathrm{d} 3}$ & $\mathrm{PRD}_{\mathrm{d} 4}$ & $\mathrm{PRD}_{\mathrm{d} 5}$ \\
\hline 2.4500 & 0.9705 & 271.7439 & 1.9671 & 2.2151 & 1.9326 & 0.3085 \\
\hline 3.3500 & 5.1952 & 117.1400 & 14.6527 & 4.6496 & 2.0179 & 0.4797 \\
\hline 4.2300 & 14.3690 & 271.7500 & 47.9929 & 6.4779 & 19.5720 & 5.4853 \\
\hline 4.9300 & 23.9059 & 446.8700 & 86.4514 & 18.5291 & 36.8588 & 8.2393 \\
\hline 5.4900 & 33.6200 & 526.0700 & 145.2222 & 45.7119 & 64.7543 & 10.8604 \\
\hline 6.4300 & 55.5288 & 671.0000 & 251.9523 & 141.1878 & 141.1803 & 16.2761 \\
\hline
\end{tabular}

Table 3: PRDS and SMR of speech signal

\begin{tabular}{|c|c|c|c|c|c|c|c|c|c|}
\hline $\mathrm{PRD}_{\text {org }}$ & $\mathrm{PRD}_{\mathrm{d} 1}$ & $\mathrm{PRD}_{\mathrm{d} 2}$ & $\mathrm{PRD}_{\mathrm{d} 3}$ & $\mathrm{PRD}_{\mathrm{d} 4}$ & $\mathrm{PRD}_{\mathrm{d} 5}$ & $\mathrm{PRD}_{\mathrm{s} 5}$ & $\mathrm{M}$ & SMR & SNR \\
\hline \multicolumn{10}{|c|}{ Thresholding 5\%, CR = 2.32 } \\
\hline 0.0992 & 0.3871 & 0.1538 & 0.0957 & 0.0415 & 0.0273 & 0.2238 & 0.1549 & 2.8333 & 3.4956 \\
\hline \multicolumn{10}{|c|}{ Thresholding $10 \%, C R=3.03$} \\
\hline 0.1433 & 0.7391 & 0.3186 & 0.1999 & 0.0860 & 0.0578 & 0.2668 & 0.2780 & 1.6631 & 0.2970 \\
\hline \multicolumn{10}{|c|}{ Thresholding 15\%, CR = 3.74} \\
\hline 0.1971 & 1.0249 & 0.4865 & 0.3300 & 0.1401 & 0.1001 & 0.2238 & 0.3842 & 0.9451 & -1.380 \\
\hline \multicolumn{10}{|c|}{ Thresholding 25\%, CR = 5.62} \\
\hline 0.3233 & 1.5187 & 0.8008 & 0.5630 & 0.2530 & 0.2053 & 0.4317 & 0.6287 & 0.0310 & -3.594 \\
\hline \multicolumn{10}{|c|}{ Thresholding 30\%, CR = 6.22} \\
\hline 0.3870 & 1.6961 & 0.9297 & 0.6702 & 0.3092 & 0.2615 & 0.5038 & 0.7284 & -0.2632 & -4.144 \\
\hline \multicolumn{10}{|c|}{ Thresholding 40\%, CR = 9.31 } \\
\hline 2.14 & 9.3790 & 4.6078 & 3.7930 & 2.0059 & 1.2811 & 2.8752 & 3.9903 & -3.6648 & -8.913 \\
\hline
\end{tabular}

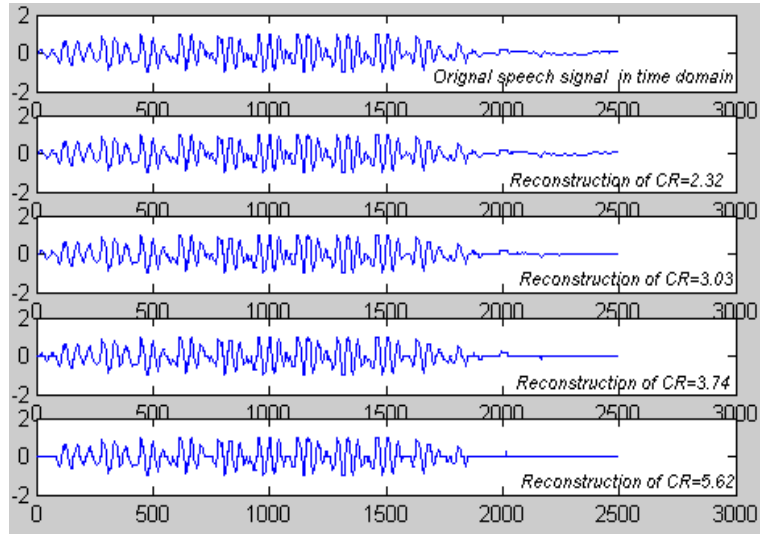

Fig. 6: The original speech signal and reconstructed signals of different acceptable compression ratios

Speech signal: For speech signal the most important reconstruction signal evaluation criteria is the objective test. Therefore, we can observe the following:

- Acceptable reconstruction speech signals of compression ratios are 5.62, 3.74, 3.03 and 2.32 (Table 3 and Fig. 6). We can see that these all signal are deformatted in $\mathrm{s}_{4}$. Therefore, we can propose that (PRDS) test is not suitable for speech signal evaluation

- $\quad$ SMR is proposed for speech reconstruction quality assessment. In Table 3 we can see that for CR 3.74 and 5.62, (SNR) (acceptable) is negative, which is a sign of high error, but SMR is positive. It means that (SMR) gives better assessment dependents on objective test

\section{CONCLUSSION}

In this research evaluation of reconstruction method is presented. The proposed method that based on wavelet transform shows good capability of deformation places detecting in the reconstruction signal. The main places of deformation exists in $\mathrm{d}_{3}, \mathrm{~d}_{4}$ or $\mathrm{s}_{4}$ sub-signals. This will guide us to study the performance of the used compression method in preserving the original information in the reconstruction signals. The presented method shows sharp results for (ECG) signal compression method evaluation.

But this method is not accurate for speech because of very sensitive intensive nature signal. The signal to noise ratio based on the relation of the energy of reconstructed signal and difference between original and reconstructed signal (DWT) sub-signals energy SMR shows better assessment than the signal to noise ratio based on the relation of the energy of reconstructed signal and difference between original and reconstructed signal energy SNR.

\section{REFERENCES}

1. Mikhled Alfaouri and K. Daqrouq, 2008. ECG signal denoising by wavelet transform thersholding. Am. J. Applied Sci., 5 (3): 276-281. 
2. Shapiro, J.M., 1993. Embedded image coding using zero trees of wavelet coefficients. IEEE Trans. Signal Process., 41 (12): 3445-3463.

3. Cuiwei, L., Z. Chongxun and T. Changfeng, 1995. Detection of ECG characteristic points using wavelet transform. IEEE Trans. Biomed. Eng., 42 (1): 1995.

4. Alfaouri Mikhled, 1997. Time-frequency analysis of non-stationary signal. AL-Balqa' J. Res. Stud., Amman-Jordan, 5 (1).

5. Knipe, J., X. Li and B. Han, 1998. An improved lattice vector quantization scheme for wavelet compression. IEEE Trans. Signal Process., 46 (1): 239-243.

6. Boulgouris, N.V., D. Tzovaras and M.G. Strintzis, 2001 Lossless image compression based on optimal prediction, adaptive lifting and conditional arithmetic coding. IEEE Trans. Image Process., 10 (1): $1-14$.
7. Alfaouri Mikhled, 1998. New algorithms for digital analysis of power intensity of non- stationary signals. Eng. J. Univ. Qatar, 11: 169-176.

8. Daqrouq, K., 2005. ECG baseline wandering reduction method using wavelet transform. Asian J. Inform. Technol. (Medwell), 4 (11).

9. Al-Qawasmi, A. and K. Daqrouq, 2006. Data compression method using zero-position coding. IPEC Conference, December 2006, pp: 18-21.

10. Zigel, Y., A. Cohen and A. Katz, 2000. The Weigted Diagnosis Distortion (WDD) measure for ECG signal compression. IEEE Trans. Biom. Eng., 47 (11): 329-343. 